\title{
Clinical Medicine Insights: Case Reports
}

\section{A Case of Severe Acute Necrotizing Pancreatitis after Administration of Sitagliptin}

\author{
Mariko Sue, Aya Yoshihara, Koji Kuboki, Naoki Hiroi and Gen Yoshino \\ Division of Diabetes, Metabolism and Endocrinology, Department of Internal Medicine (Omori), \\ Toho University School of Medicine, Tokyo, Japan. Corresponding author email: n-hiroi@med.toho-u.ac.jp
}

\begin{abstract}
A 55-year-old Japanese man with a 3-year history of type 2 diabetes mellitus was admitted to our hospital for upper abdominal pain. Control of diabetes mellitus was good with voglibose and metformin, with sitagliptin added to this regimen 8 months prior. His pancreatic enzyme levels were elevated, and abdominal computed tomography (CT) showed diffuse pancreatic swelling with fluid accumulation and ascites of CT grade 3. The patient was diagnosed with severe acute pancreatitis. There were no obvious causes for pancreatitis except the recently administered sitagliptin. Since incretin-related drugs entered the market, the number of incretin-related drugs prescriptions rapidly increased and so did the incidence of pancreatitis. There are several reports suggesting the correlation between incretin-related drugs and pancreatitis, such as a report based on data obtained from the United States Food and Drug Administration (FDA) which revealed a significant correlation between the administration of exenatide or sitagliptin and pancreatitis. However, there also is a report that denied the evidence for such in a large cohort study. The relation between incretin based drugs and pancreatitis is still controversial.
\end{abstract}

Keywords: diabetes mellitus, DPP-4 inhibitor, sitagliptin, pancreatitis

Clinical Medicine Insights: Case Reports 2013:6 23-27

doi: $10.4137 /$ CCRep.S10856

This article is available from http://www.la-press.com.

(C) the author(s), publisher and licensee Libertas Academica Ltd.

This is an open access article. Unrestricted non-commercial use is permitted provided the original work is properly cited. 


\section{Introduction}

Dipeptidyl peptidase-4 (DPP-4) inhibitors and glucagon-like peptide-1 (GLP-1) analogs are incretin-based drugs; these drugs have found widespread use as a new class of anti-hyperglycemic agents effective for treating diabetes mellitus. GLP-1 is reported to slow food absorption, improve insulin production by the pancreas, and increase beta cell mass, whereas DPP-4 inhibitors act by delaying the breakdown of GLP-1. Both types of agents lower glucose levels without weight gain and with a reduced risk of hypoglycemia, representing clear advantages over other glucose-lowering agents. ${ }^{1}$ These beneficial aspects in addition to ease of use, particularly in the case of the DPP-4 inhibitors, has accelerated their worldwide use. As these are newly developed drugs, their long-term side effects are still unknown. However, concerns regarding the association between the use of these drugs and pancreatitis and pancreatic or thyroid cancers have increased, and need to be investigated.

\section{Case Report}

A 55-year-old Japanese man with type 2 diabetes mellitus was admitted to our hospital with a 24 hour history of upper abdominal pain. At a previous visit to another hospital, he had been diagnosed with gastric ulcer and prescribed a histamine $\mathrm{H} 2$ blocker. However, his symptoms worsened, with increased back pain and frequent vomiting. At the time of the diagnosis, his type 2 diabetes mellitus had been controlled with $0.6 \mathrm{mg}$ of voglibose and $500 \mathrm{mg}$ of metformin per day, both initiated 3 years before. In addition with that, $50 \mathrm{mg}$ of sitagliptin per day had been added to his treatment regimen 8 months earlier, and diabetes mellitus was in good control with $\mathrm{HbA}_{1 \mathrm{c}}$ below $6.5 \%$. His latest fasting glucose and glycosylated hemoglobin levels were $111 \mathrm{mg} / \mathrm{dL}$ and $6.2 \%$, respectively. He had no history of chronic pancreatitis, pancreatic tumor, hypercalcemia, or habitual alcohol use. Hyperlipidemia had also been diagnosed at the same time of the diabetes mellitus diagnosis, however was well controlled with $10 \mathrm{mg}$ of atorvastatin.

On physical examination, his height, weight, and body mass index were found to be $175 \mathrm{~cm}, 77.2 \mathrm{~kg}$, and $25.2 \mathrm{~kg} / \mathrm{m}^{2}$, respectively. His body temperature, blood pressure, and heart rate were $38.1{ }^{\circ} \mathrm{C}$,
172/88 $\mathrm{mmHg}$, and 98 beats/min, respectively. Upper abdominal tenderness was observed without mass or rigidity. C-reactive protein level was $0.1 \mathrm{mg} / \mathrm{dL}$, and white blood cell count was $21800 / \mu \mathrm{L}$. His pancreatic and liver enzyme level were elevated at the hospitalization (amylase level, $3581 \mathrm{IU} / \mathrm{L}$; pancreatic amylase level, $3435 \mathrm{IU} / \mathrm{L}$; elastase-1 level, $6749 \mathrm{ng} / \mathrm{dL}$, aspartate aminotransferase level, $266 \mathrm{IU} / \mathrm{L}$; and alanine aminotransferase level, 137 IU/L). Abdominal computed tomography (CT) revealed elevated adipose tissue concentration in the pancreas, duodenum, and transverse colon. Ascites was present on the liver surface, abdominopelvic cavity, peripancreatic area, and posterior pararenal extraperitoneal space; furthermore, a gallstone was detected (Fig. 1A). The Acute Physiology, Age, and Chronic Health Evaluation
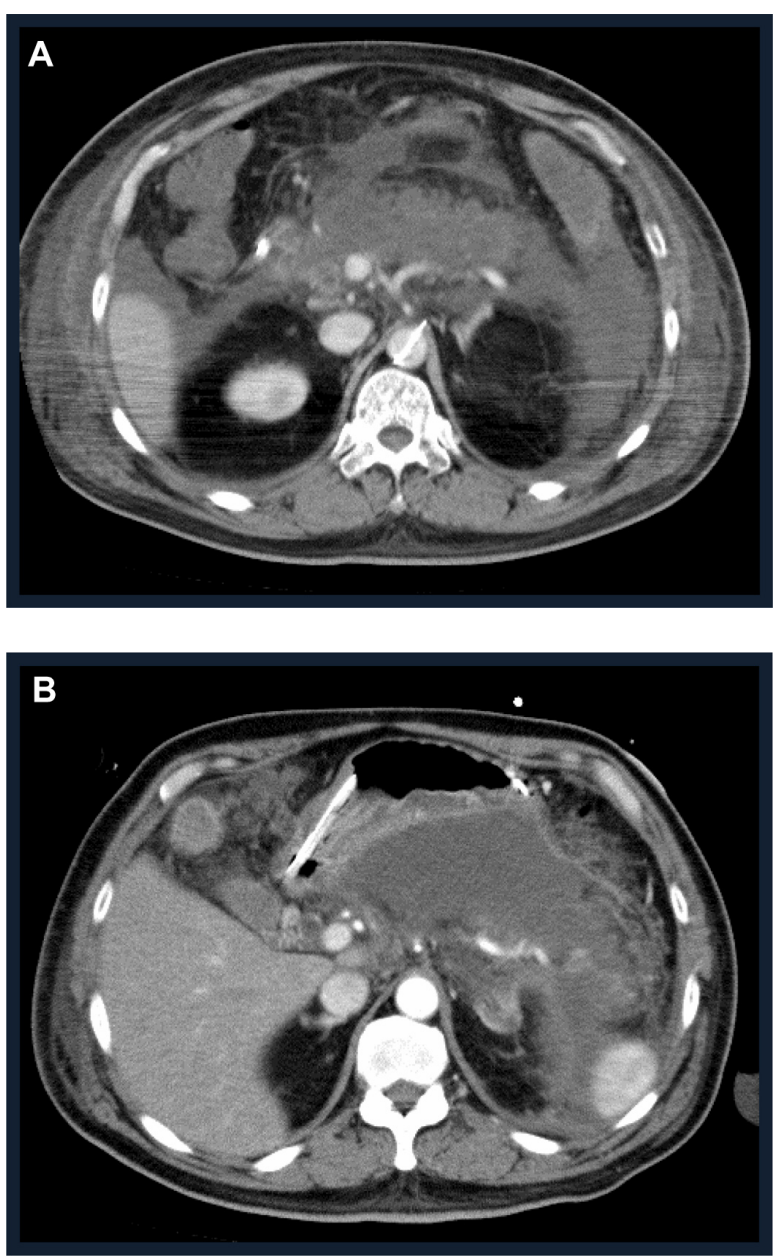

Figure 1. A computed tomography (CT) scan of the abdomen on admission revealed peripancreatic inflammatory changes, with ascites on liver surface, abdominopelic cavity, peripancreaticposterior pararenal extraperitoneal space $(\mathbf{A})$. A CT scan of the abdomen on 13th day after hospitalization revealed poorly enhanced areas in the body and tail of the pancreas consistent with necrosis and cyst formation (B). 
(APACHE) score was 6 points, Systemic Inflammatory Response Syndrome (SIRS) score was 3, and CT scan severity index was grade 3 . He was diagnosed with severe acute pancreatitis.

\section{Clinical Course}

His condition was complicated by severe systemic inflammation, disseminated intravascular coagulation, and respiratory insufficiency, which required treatment comprising intravenous fluid therapy, administration of antibiotics and pancreatic enzyme inhibitors systemically and via an arterial catheter, use of a respirator, and continuous hemodiafiltration in the intensive care unit. Three days after admission, total bilirubin increased. However, CT revealed no signs of common bile duct dilatation, choledocholithiasis, or gallstone incarceration. Hyperbilirubinemia improved a few days after treatment for pancreatitis, and there was no recurrence of pancreatitis or hyperbilirubinemia during hospitalization; therefore, we eliminated pancreatitis by gallstone.

Extensive inflammation and necrosis were observed, and the pancreatic cyst was resistant to therapy, requiring nearly 3 months of daily drainage and weekly necrectomy (Fig. 1B). On day 87 after admission, an oral diet was started. In spite of the improvement in pancreatitis, his insulin levels gradually decreased, with serum and urine C-peptide concentrations of $0.52 \mathrm{ng} / \mathrm{mL}$ and $38.6 \mu \mathrm{g} /$ day, respectively. The delta C-peptide level after glucagon loading was $0.31 \mathrm{ng} / \mathrm{mL}$ (serum C-peptide levels before and $6 \mathrm{~min}$ after glucagon stimulation were 0.38 and $0.69 \mathrm{mg} / \mathrm{dL}$, respectively). At that time, a diagnosis of pancreatic diabetes was made. His diabetes mellitus was well controlled with a total insulin injection of 28 units per day. He was discharged from hospital 111 days after admission. There is no recurrence of pancreatitis for 14 months after discontinuation of sitagliptin.

\section{Discussion}

Acute pancreatitis is known to be fatal, with a mortality rate of nearly $10 \%$ if severe disease is not diagnosed and if appropriate treatments are not initiated immediately. ${ }^{2}$ Even in cases in which diagnosis and treatment are rapid, severe pancreatitis can prove life threatening. Various causes for pancreatitis include alcohol consumption, gallstones, idiopathic chronic pancreatitis, endoscopic retrograde cholangiopancreatography or endoscopic sphincterotomy, trauma, malignancy, autoimmune hypercalcemia, hyperlipidemia, or certain drugs, of which alcohol and gallstone are the 2 primary causes in Japan. ${ }^{3}$ Our patient did not have a history of alcohol intake within the few months prior to hospitalization. In addition, he had no history of a medical condition that could cause acute pancreatitis, except hyperlipidemia, which was well controlled with atorvastatin for the past 3 years. Because abdominal CT revealed gallstones and bilirubin was elevated after hospitalization, we first suspected gallstone pancreatitis. However, neither CT nor ultrasound imaging indicated biliary ductal dilatation involving the common bile duct. Hyperbilirubinemia improved only a few days after treatment for pancreatitis had begun, suggesting that bilirubinemia might have developed as a result of the spread of inflammation from pancreatitis. In addition, there was no recurrence of pancreatitis for 14 months without gall stone treatment.

Medications are also known to cause pancreatitis, with a frequency of $1.4 \%$ to $2.0 \%{ }^{4}$ There are several reports describing pancreatitis associated with metformin and atorvastatin. In the Japanese guidelines for pancreatitis published in 2010, metformin and atorvastatin are not included. However, as one of the HMG-CoA reductase inhibitors group, pravastatin is classified within class I (medications in which at least 1 case report described a recurrence of acute pancreatitis with a rechallenge with the drug). ${ }^{5}$ An exhaustive search of the literature revealed only 6 and 5 cases of pancreatitis associated with atorvastatin ${ }^{6-11}$ and metformin, respectively. ${ }^{12-16}$ In most of these cases, drugs were administered for less than a year. With the increased use of incretin-related drugs, a few cases of acute pancreatitis that had been suspected to be related with DPP-4 inhibitors or GLP-1 agonists have been reported. To the best of our knowledge, only 4 cases of pancreatitis related to sitagliptin and vildagliptin have been reported (Table 1). One patient was treated with sitagliptin for 8 weeks, ${ }^{17} 2$ other patients were switched from sitagliptin to vildagliptin 2 weeks or 6 months before; ${ }^{18,19}$ and 1 patient received combined therapy of sitagliptin and exenatide for a few weeks. ${ }^{20}$ Raschi et al reported a significant correlation between GLP-1, DPP-4 inhibitors, and pancreatitis, on the basis of the information in the FDA database. ${ }^{21}$ Among these drugs, sitagliptin and exenatide are reported to have a 
Table 1. Reports of pancreatitis cases related with DPP-4 inhibitors.

\begin{tabular}{lllllllll}
\hline Case & Age & Sex & DPP-4 inhibitor & GLP-1 & Duration & Other OHA & CT grade & Ref. \\
\hline 1 & 53 & F & Sitagliptin & N/A & 8 weeks & Metformin, gliclazide & 1 \\
2 & 76 & F & Sitagliptin & Exenatide & Few weeks & N/A & 17 & $3-4$ \\
3 & 74 & M & Sitagliptin $\rightarrow$ vildagliptin & N/A & 2 weeks & Glimepirid, pioglitazone & 1 & 18 \\
4 & 61 & F & Sitagliptin $\rightarrow$ vildagliptin & N/A & 5 weeks & Metformin & 19 \\
\hline
\end{tabular}

strong correlation with pancreatitis. The average duration from administration of sitagliptin and exenatide to onset of pancreatitis was 444 days and 515 days, respectively, which was similar to that in our case in which pancreatitis was diagnosed 8 months after the administration of sitagliptin.

Animal studies showed that GLP-1-based treatments induced pancreatitis in a rat model of type 2 diabetes, with medications such as sitagliptin increasing pancreatic ductal replication and acinar-to-ductal metaplasia. ${ }^{22-24}$ Exenatide has also been found to induce pancreatic acinar inflammation, with abnormal acinar cells and a high frequency of cell death..$^{25}$ Further, in type 2 diabetes patients, pancreatitis is 6 -fold more likely to be reported in association with sitagliptin or exenatide than with other therapies. ${ }^{23}$ These drugs are also suggested to be correlated to pancreatitis and increased risks for pancreatic and thyroid cancers. However, Dore et al. reported that no evidence was found for an increased incidence of pancreatitis in a large cohort of patients treated with either exenatide or sitagliptin, compared to those treated with metformin or glyburide. ${ }^{26}$

\section{Conclusion}

There are many case reports and studies suggesting that GLP-1 agonists and DPP-4 inhibitors, which are widely used and promoted for the treatment of type 2 diabetes, could have serious accidental and unexpected side effects. We reported a case of acute necrotic pancreatitis in which a DPP-4 inhibitor had been administered 8 months before. While the DPP-4 inhibitor was suspected to be the cause of pancreatitis, the correlation between incretin-based drugs and pancreatitis remains controversial.

\section{Author Contributions}

Conceived and designed the experiments: MS. Analysed the data: MS. Wrote the first draft of the manuscript: MS. Contributed to the writing of the manuscript: MS, NH. Agree with the manuscript results and conclusions: MS, NH, AY, KK, GY. Jointly developed the structure and arguments for the paper: MS, NH. Made critical revisions and approved final version: MS, NH. All authors reviewed and approved of the final manuscript.

\section{Funding}

Author(s) disclose no funding sources.

\section{Competing Interests}

Author(s) disclose no potential conflicts of interest.

\section{Disclosures and Ethics}

As a requirement of publication author(s) have provided to the publisher signed confirmation of compliance with legal and ethical obligations including but not limited to the following: authorship and contributorship, conflicts of interest, privacy and confidentiality and (where applicable) protection of human and animal research subjects. The authors have read and confirmed their agreement with the ICMJE authorship and conflict of interest criteria. The authors have also confirmed that this article is unique and not under consideration or published in any other publication, and that they have permission from rights holders to reproduce any copyrighted material. Any disclosures are made in this section. The external blind peer reviewers report no conflicts of interest.

\section{References}

1. Scheen AJ. Dipeptidylpeptidase-4 (DPP-4) inhibitors are favorable to glucagon-like peptide-1 (GLP-1) receptor agonists: yes. Eur J Intern Med. 2012;23(2):126-31.

2. Mayumi T, Ura H, Arata S, et al. Working Group for the Practical Guidelines for Acute Pancreatitis. Japanese Society of Emergency Abdominal Medicine. J Hepatobiliary Pancreat Surg. 2002;9(4):413-22.

3. Sekimoto M, Takada T, Kawarada Y, et al. Japanese guidelines for the management of acute pancreatitis: epidemiology, etiology, natural history, and outcome predictors in acute pancreatitis. $J$ Hepatobiliary Pancreat Surg. 2006;13(1):10-24. 
4. McArthur KE. Review article: drug-induced pancreatitis. Aliment Pharmacol Ther. 1996;10(1):23-38.

5. Takada T, Kawarada Y, Hirata K, et al. Japanese guidelines for the management of acute pancreatitis: cutting-edge information. $J$ Hepatobiliary Pancreat Surg. 2006;13(1):2-6.

6. Deshpande PR, Khera K, Thunga G, Hande M, Gouda ST, Nagappa AN Atorvastatin-induced acute pancreatitis. J Pharmacol Pharmacother. 2011; 2(1):40-2.

7. Prajapati S, Shan S, Desai C, Desai M, Dikshit RK. Atorvastatin-induced pancreatitis. Indian J Pharmacol. 2010;42(5):324-5.

8. Kanbay M, Sekuk H, Yilmaz U, Gur G, Boyacioglu S. Acute pancreatitis associated with combined lisinopril and atorvastatin therapy. Dig Dis. 2005;23(1):92-4.

9. Singh S, Nautiyal A, Dolan JG. Recurrent acute pancreatitis possibly induced by atorvastatin and rosuvastatin. Is statin induced pancreatitis a class effect? JOP. 2004;5(6):502-4.

10. Miltiadous G, Anthopoulou A, Elisaf M. Acute pancreatitis possibly associated with combined salicylate and atorvastatin therapy. JOP. 2003;4(1): $20-1$.

11. Belaiche G, Ley G, Slama JL. Acute pancreatitis associated with atorvastatin therapy. Gastroenterol Clin Biol. 2000;24(4):471-2.

12. Molina Infante J,PrietoBermejoAB,PerezGallardoB,FernandezBermejo M. Toxic metformin-associated acute pancreatitis without kidney failure. Med Clin (Barc). 2008;131(13):519.

13. Audia P, Feinfeld DA, Dubrow A, Winchester JF. Metformin-induced lactic acidosis and acute pancreatitis precipitated by diuretic, celecoxib, and candesartan-associated acute kidney dysfunction. Clin Toxicol (Phila). 2008; 46(2):164-6.

14. Fimognari FL, Corsonello A, Pastorell R, Antonelli-Incalzi R. Metformin-induced pancreatitis: a possible adverse drug effect during acute renal failure. Diabetes Care. 2006;29(5):1183.

15. Mallick S. Metformin induced acute pancreatitis precipitated by renal failure. Postgrad Med J. 2004;80(942):239-40.

16. Ben MH, Thabet H, Zaghdoudi I, Amamou M. Metformin associated acute pancreatitis. Ven Hum Toxicol. 2002;44(1):47-8.
17. Garg R, Hussey C, Ibrahim S. Pancreatitis associated with the use of sitagliptin and orlistat combination: a case report. Diabet Med. 2010;27(4):485-6.

18. Iyer SN, Drake AJ 3rd, West RL, Mendez CE, Tanenberg RJ. Case report of acute necrotizing pancreatitis associated with combination treatment of sitagliptin and exenatide. Endocr Pract. 2012;18(1):e10-3.

19. Matsushita M, Yamada Y, Katou Y, Nakano M, Yamada S. Oral presentation in 582nd Japanese society of internal medicine, Kanto region. Unpublished observation.

20. Girgis CM, Champion BL. Vildagliptin-induced acute pancreatitis. Endocr pract. 2011;17(3):e48-50.

21. Raschi E, Piccinni C, Poluzzi E, Marchesini F, De Ponti F. The association of pancreatitis with antidiabetic drug use: gaining insight through the FDA pharmacovigilance database. Acta Diabetol. Oct 19, 2011. [Epub ahead of print.]

22. Matveyenko AV, Dry S, Cox HI, et al. Beneficial endocrine but adverse exocrine effects of sitagliptin in the human islet amyloid polypeptide transgenic rat model of type 2 diabetes: interactions with metformin. Diabetes. 2009;58(7):1604-15.

23. Elashoff M, Matveyenko AV, Gier B, Elashoff R, Butler PC. Pancreatitis, pancreatic, and thyroid cancer with glucagon-like peptide-1-based therapies. Gastroenterology. 2011;141(1):150-6.

24. Koehler JA, Baggio LL, Lamont BJ, Ali S, Drucker DJ. Glucagon-like peptide-1 receptor activation modulates pancreatitis-associated gene expression but does not modify the susceptibility to experimental pancreatitis in mice. Diabetes. 2009;58(9):2148-61.

25. Nachnani JS, Bulchandani DG, Nookala A, et al. Biochemical and histological effects of exendin-4 (exenatide) on the rat pancreas. Diabetologia. 2010;53(1):153-9.

26. Dore DD, Seeger JD, Arnold Chan K. Use of a claims-based active drug safety surveillance system to assess the risk of acute pancreatitis with exenatide or sitagliptin compared to metformin or glyburide. Curr Med Res Opin. 2009;25(4):1019-27. 